\title{
DEFINITIONS FOR A CLASS OF PLANE QUASICONFORMAL MAPPINGS
}

\author{
F. W. GEHRING')
}

Dedicated to Professor K. Noshiro on his sixtieth birthday

1. Introduction. This report is a survey of some of the many different ways of characterizing a class of plane quasiconformal mappings. This class was considered by Ahlfors [4] in his treatment of the Teichmüller problem, and it has been studied rather extensively in the last ten years.

In order to simplify notation, we shall consider only mappings between finite plane domains. Hence in what follows, $D$ and $D^{\prime}$ will denote domains in the finite plane $R^{2}$, and $f$ will denote an orientation preserving homeomorphism of $D$ onto $D^{\prime}$. Next for each quantity $\delta$ associated with $D$, such as a point, subset, or family of subsets, we shall let $\delta^{\prime}$ denote its image under $f$. Finally given $1 \leq K<\infty$, we let $Q_{K}$ denote the class of all $K$-quasiconformal homeomorphisms $f$, and $Q$ the union of the classes $Q_{K}$ for $1 \leq K<\infty$.

In the following sections we first list nine equivalent definitions for the class $Q_{K}$. Then we give three additional definitions for the class $Q$ in the special case where $D=D^{\prime}=R^{2}$. Finally we indicate where one may find proofs for the equivalence of these definitions.

2. Closure of the class of differentiable quasiconformal mappings. Given $1 \leq K<\infty$, we let $Q_{K}^{*}$ denote the class of diffeomorphisms $f$ which map each infinitesimal circle in $D$ onto an infinitesimal ellipse with axis ratio bounded above by $K$. Then $Q_{K}^{*}$ is the class of $K$-quasiconformal mappings studied by Grötzsch and Teichmüller, and it is well known that many important extremal problems for mappings in $Q_{K}^{*}$ have solutions in $Q_{K}^{*}$. This fact is, at first, rather surprising since $Q_{K}^{*}$ is obviously not closed under uniform convergence

Received June 22, 1966.

1) This paper is the manuscript of a talk given at the Conference on Quasiconformal Mappings, Moduli, and Discontinuous Groups held in New Orleans in May 1965. : The research for this report was supported in part by the Air Force, Grant AFOSR-393.63. 
on compact sets. We are thus led to our first characterization for the class $Q_{K}$ (See [18].).

DEFINITION 1. $f \in Q_{K}$ if and only if there exists a sequence of mappings $f_{n} \in Q_{K}^{*}$ of $D_{n}$ onto $D_{n}^{\prime}$ such that $f_{n} \rightarrow f$ uniformly on each compact subset of $D$.

3. Analytic definition. We say that $f$ is absolutely continuous on lines or simply $A C L$ in $D$ if for each disk $U$ with $\bar{U} \subset D, f$ is absolutely continuous on almost all line segments in $U$ which are parallel to one of the coordinate axes. For such an $f$, the complex derivatives $f_{z}=\frac{1}{2}\left(f_{x}-i f_{y}\right)$ and $f_{\bar{z}}=\frac{1}{2}\left(f_{x}+i f_{y}\right)$ exist a.e. in $D$, and it follows that $f$ is differentiable a.e. in $D$ [14]. . The analytic definition for the class $Q_{K}$ can then be stated as follows (See [8], [14], [20], and [23].).

DEFINITION 2. $f \in Q_{K}$ if and only if $f$ is $A C L$ in $D$ and $\left|f_{z}\right|+\left|f_{\bar{z}}\right| \leqq K\left(\left|f_{z}\right|-\left|f_{\bar{z}}\right|\right)$ a.e. in $D$.

If $f \in Q_{K}$ by Definition 2, then the fact that $f$ is a homeomorphism implies that $f_{z}$ and $f_{\bar{z}}$ are locally $L^{2}$-integrable. Hence the following is an alternative formulation of Definition 2 (See [7] and [9].).

DEFINITION 2'. $f \in Q_{K}$ if and only if $f$ has $L^{2}$ derivatives and satisfies $f_{\bar{z}}=\mu f_{z}$ a.e. in $D$, where $|\mu| \leqq \frac{K-1}{K+1}$.

4. Configurations with one conformal invariant. Let $\Sigma$ be a configuration consisting of a domain bounded by $m$ Jordan curves, together with $n$ boundary points and $p$ interior points distinguished. Then the four cases where $\Sigma$ has precisely one conformal invariant or module are as follows (See p. 88 in [3].).

$$
\begin{array}{ll}
1^{\circ} . & m=2, n=0, p=0, \\
2^{\circ} . & m=1, n=4, p=0, \\
3^{\circ} . & m=1, n=2, p=1, \\
4^{\circ} . & m=1, n=0, p=2 .
\end{array}
$$

In the first case, $\Sigma$ is a ring $R$ and an obvious conformal invariant is $\bmod R$, the logarithm of the radius ratio of any conformally equivalent annulus. In the second case, $\Sigma$ is a quadrilateral $\Omega$ and a convenient invariant is $\bmod \Omega$, the side ratio of any conformally equivalent rectangle. In the third case, $\Sigma$ is a Jordan domain $\Delta$ with a boundary arc $\alpha$ and an interior point $z_{0}$ distinguished, 
and we may take as a conformal invariant the harmonic measure $\omega\left(\alpha ; z_{0}\right)$ of $\alpha$ at $z_{0}$. Finally in the fourth case, $\Sigma$ is a Jordan domain $\Delta$ with two interior points $z_{1}$ and $z_{2}$ distinguished, and the hyperbolic distance $h\left(\Delta ; z_{1}, z_{2}\right)$ between $z_{1}$ and $z_{2}$ is a conformal invariant.

5. Rings. Each of the above configurations may be used to characterize the class $Q_{K}$. In particular, since many distortion properties for quasiconformal mappings can be obtained from studying how the moduli of certain extremal rings are changed under the mappings, the following characterization is of some interest (See [15] and [24].).

Definition 3. $f \in Q_{K}$ if and only if $\bmod R^{\prime} \leq K \bmod R$ for all rings $R$ with $\bar{R} \subset D$.

It is possible to associate with each ring in $n$-space, $n \geq 3$, a modulus or conformal capacity which is invariant with respect to Möbius transformations [19], and hence Definition 3 suggests a way of defining quasiconformality in higher dimensions [12].

6. Quadrilaterals. Historically, the first definition given for the class $Q_{K}$ is the following due to Ahlfors [4] (See also [22].).

Definition 4, $f \in Q_{K}$ if and only if $\bmod \Omega^{\prime} \leq K \bmod \Omega$ for all quadrilaterals $\Omega$ with $\bar{\Omega} \subset D$.

There are several variants of this definition where the quadrilaterals are restricted to various subclasses. For example, one can show that $f \in Q_{\pi}$ if $\bmod \Omega^{\prime} \leq K \bmod \Omega$ for all quadrilaterals $\Omega$ which are rectangles [15]. It is also true that $f \in Q_{K}$ if $\bmod \Omega^{\prime} \leq K \bmod \Omega$ for all quadrilaterals $\Omega$ with $\bmod \Omega=1$ [17]. On the other hand, it is not known if $f \in Q_{K}$ provided that $\bmod \Omega^{\prime} \leq K \bmod \Omega$ for all quadrilaterals $\Omega$ which are squares, that is for all $\Omega$ which are rectangles with $\bmod \Omega=1$.

7. Harmonic and hyperbolic measures. For $0<r<1$, let $\mu(r)$ denote the modulus of the ring bounded by the unit circle $|z|=1$ and the segment $0 \leq x \leq r$, $y=0$. We then can characterize the class $Q_{K}$ by means of the remaining two configurations $\Sigma$, with just one conformal invariant, as follows (See [17].).

Definition 5. $f \in Q_{K}$ if and only if for each Jordan domain $\Delta$ with $\bar{\Delta} \subset D$, 


$$
\mu\left(\sin \frac{\pi}{2} \omega\left(\alpha^{\prime} ; z_{0}^{\prime}\right)\right) \leq K \mu\left(\sin \frac{\pi}{2} \omega\left(\alpha ; z_{0}\right)\right)
$$

for all $\alpha \subset \partial \Delta$ and $z_{0} \in \Delta$.

Definition 6. $f \in Q_{K}$ if and only if for each Jordan domain $\Delta$ with $\bar{\Delta} \subset D$,

$$
\mu\left(e^{-2 h\left(\Delta^{\prime} ; z_{1}^{\prime}, z_{2}\right)}\right) \leq K \mu\left(e^{-2 h\left(\Delta ; z_{1}, z_{2}\right)}\right)
$$

for all $z_{1}, z_{2} \in \Delta$.

8. Extremal lengths. Let $\Gamma$ be a family of arcs or Jordan curves in the finite plane $R^{2}$, and let $F(\Gamma)$ denote the family of functions $\rho$ which are nonnegative and Borel measurable in $R^{2}$ and for which

$$
\int_{r} \rho|d z| \geq 1
$$

for each locally rectifiable $r \in \Gamma$. The modulus of $\Gamma$ is defined as

$$
M(\Gamma)=\inf _{\rho} \iint_{R^{2}} \rho^{2} d x d y, \quad \rho \in F(\Gamma),
$$

and the extremal length is given by $\lambda(\Gamma)=\frac{1}{M(\Gamma)}$. Because each of the conformal invariants mentioned above can be expressed in terms of extremal lengths, [3] and [16], the following characterization is of interest [27].

DEFinition 7. $f \in Q_{K}$ if and only if $\lambda\left(\Gamma^{\nu}\right) \leq K \lambda(\Gamma)$ for all curve families $\Gamma \subset D$.

This definition suggests another way of defining quasiconformality in higher dimensions [27].

We say that $f$ preserves the curve families of extremal length zero if for each family $\Gamma \subset D, \lambda\left(\Gamma^{\prime}\right)=0$ if and only if $\lambda(\Gamma)=0$. From [25] and [27] we obtain the following characterization for the class $Q$.

Definition $7^{\prime} . f \in Q$ if and only if $f$ preserves the curve families of extremal length zero.

9. Distortion of infinitesimal circles. Given a homeomorphism $f$, we set

$$
H(z)=\lim \sup _{r \rightarrow 0} \frac{\max _{|h|=r}|f(z+h)-f(z)|}{\min _{|h|=r}|f(z+h)-f(z)|}
$$

for each $z \in D$. Then $f \in Q_{K}^{*}$ if and only if $f$ is a diffeomorphism with $H \leq K$ 
in $D$. We have the following analogous characterization for $Q_{K}$ (See [11] and [21].).

Definition 8. $f \in Q_{K}$ if and only if $H$ is bounded in $D$ and $H \leq K$ a.e. in D.

There exists a homeomorphism $f \in Q_{K}$ for which $H=K^{2}$ on a set of Hausdorff dimension 2 [11]. Hence, except when $K=1$, it is not possible to replace the two conditions in Definition 8 by the single requirement that $H \leq K$ at all points of $D$. However, for the class $Q$ we obviously have the following characterization.

Definition $8^{\prime} . f \in Q$ if and only if $H$ is bounded in $D$.

Again these definitions suggest how one may define analogous classes in higher dimensions.

10. Distortion of angles. A conformal mapping is an angle preserving diffeomorphism. There is an obvious modification of this characterization for $Q_{K}^{*}$. On the other hand, it is not immediately clear how to proceed for $Q_{K}$. This is because a mapping $f \in Q_{K}$ may have an exceptional set $E$ of zero measure at which it is not differentiable; thus an angle with vertex in $E$ may be carried by $f$ onto a pair of arcs with no tangents at their common endpoint. In order to circumvent this difficulty, given two arcs $\gamma_{1}$ and $\gamma_{2}$ which meet only at a common endpoint $z_{0}$, we let

$$
A\left(\gamma_{1}, \gamma_{2}\right)=\liminf _{z_{1}, z_{2} \rightarrow z_{0}} 2 \arcsin \left(\frac{\left|z_{1}-z_{2}\right|}{\left|z_{1}-z_{0}\right|+\left|z_{0}-z_{2}\right|}\right), \quad z_{i} \in \gamma_{i} .
$$

This is a kind of angular measure for the topological angle formed by $\gamma_{1}$ and $r_{2}$ at $z_{0}$, and it reduces to the smaller of the two angles formed by $\gamma_{1}$ and $\gamma_{2}$ when these arcs are segments. We then have the following characterization for the class $Q_{K}$ (See [2].).

DEFINITION 9. $f \in Q_{K}$ if and only if the following two conditions are satisfied.

(i) For all $z_{0} \in D$ and all segments $\gamma_{1}, \gamma_{2} \subset D$ which form an angle at $z_{0}$, $A\left(r_{1}^{\prime}, r_{2}^{\prime}\right)>0$.

(ii) For almost all $z_{0} \in D$ and all segments $\gamma_{1}, \gamma_{2} \subset D$ which form an angle at $z_{0}, A\left(r_{1}^{\prime}, r_{2}^{\prime}\right) \geq \frac{1}{K} A\left(r_{1}, r_{2}\right)$.

Except when $K=1$, one cannot replace conditions (i) and (ii) in Definition 
9 by the single requirement that $A\left(r_{1}^{\prime}, r_{2}^{\prime}\right) \geq \frac{1}{K} A\left(\gamma_{1}, r_{2}\right)$ for all $z_{0} \in D$ and all segments $\gamma_{1}, \gamma_{2} \subset D$ which form an angle at $z_{0}$.

There are, of course, other ways to assign an angular measure to a topological angle. For a quite different method and an analogous characterization of the class $Q_{K}$, see [26] (See also [1].).

11. Extension property. Suppose now that $D=D^{\prime}=R^{2}$. Then $f$ clearly can be extended to be a homeomorphism $\tilde{f}$ of the finite 3 -space $R^{3}$ onto itself. Given any such $\tilde{f}$, we set

$$
\tilde{H}(x)=\limsup _{r \rightarrow 0} \frac{\max _{|h|=r}|\tilde{f}(x+h)-\tilde{f}(x)|}{\min _{|h|=r}|\tilde{f}(x+h)-\tilde{f}(x)|}
$$

for each $x \in R^{3}$, and following Definition $8^{\prime}$ we say that $\tilde{f} \in \widetilde{Q}$ if and only if $\tilde{H}$ is bounded in $R^{3}$. $\widetilde{Q}$ is then the class of quasiconformal mappings of $R^{3}$ onto itself, and from Theorem 10 of [12] and a recent extension theorem due to Ahlfors [6], we obtain the following characterization for the class $Q$.

Definition 10. If $D=D^{\prime}=R^{2}$, then $f \in Q$ if and only if $f$ has an extension $\tilde{f} \in \bar{Q}$.

12. Compactness property. Beurling and Ahlfors obtained in [10] two characterizations for the class of homeomorphisms of the real line $R^{1}$ onto itself which can be extended to be quasiconformal mappings of $R^{2}$ onto itself. The first of these involves a symmetry condition, the so-called $\rho$-condition, while the second makes use of a compactness condition. Definition 10 suggests that there might be two analogous characterizations for the class $Q$ in the special case where $D=D^{\prime}=R^{2}$. This is indeed true, and it is not difficult to see that Definition $8^{\prime}$ is really a localized 2-dimensional form of the $\rho$-condition characterization. For the other characterization, given a family $F$ of homeomorphisms of $R^{2}$ onto itself, we say. that $F$ satisfies the compactness condition $(A)$ if each sequence of mappings $f_{n} \in F$, which leave the points 0 and 1 fixed, contains a subsequence of mappings which converge to a homeomorphism, uniformly on each compact set in $R^{2}$. Then if we let $S$ and $T$ denote arbitrary similarity mappings, the 2-dimensional analogue of the Beurling-Ahlfors compactness characterization can be stated as follows (See Corollary 8 of [12].). 
Definition 11. If $D=D^{\prime}=R^{2}$, then $f \in Q$ if and only if the family of all mappings of the form $S \circ f \circ T$ satisfies the compactness condition $(A)$.

13. Quasiconformal circles. Given a Jordan curve $C \subset R^{2}$, we let

$$
k(C)=\sup \frac{\left|z_{1}-z_{2}\right|\left|z_{3}-z_{4}\right|+\left|z_{2}-z_{3}\right|\left|z_{4}-z_{1}\right|}{\left|z_{1}-z_{3}\right|\left|z_{2}-z_{4}\right|},
$$

where the supremum is taken over all ordered quadruples of points $z_{1}, z_{2}, z_{\hat{\imath}}, z_{1} \in C$. Then it is not difficult to see that $1 \leq k(C) \leq \infty$ and that $k(C)=1$ if and only if $C$ is a circle. Ahlfors has also shown [5] that $C$ is a quasiconformal circle, that is the image of a circle under a quasiconformal mapping of $R^{2}$ onto itself, if and only if $k(C)<\infty$. We are thus led to our final characterization of the class $Q$ (Compare with Lemma 4 of [13].).

Definition 12. If $D=D^{\prime}=R^{2}$, then $f \in Q$ if and only if $\sup k\left(C^{\prime}\right)<\infty$, where the supremum is taken over all circles $C \subset R^{2}$.

14. Equivalence of these definitions. Proofs for the equivalence of Definitions $1,2,3,4,7$, and 8 can be found either in the references already quoted or in the recent book by Lehto and Virtanen [18]. The equivalence of Definitions 5 and 6 and of Definition 9 with the above is proved in [17] and in [2], respectively. That Definitions 10 and 11 yield the same class $Q$ when $D=D^{\prime}=R^{2}$ follows from [6] and Theorem 10 of [12] and from the plane version of Corollary 8 of [12], respectively. Finally we sketch a proof to show that the characterization of Definition 12 is equivalent to the others.

We show first that if $D=D^{\prime}=R^{2}$ and if $f \in Q_{K}$, then

$$
k\left(C^{\prime}\right) \leq \csc \left(2 \arcsin \mu^{-1}\left(\frac{K \pi}{2}\right)\right)
$$

for all circles $C \subset R^{2}$, where $\mu^{-1}(r)$ is the inverse of the function $\mu(r)$ defined in section 7. For this choose a circle $C \subset R^{2}$, let $z_{1}^{\prime}, z_{2}^{\prime}, z_{3}^{\prime}, z_{4}^{\prime}$ be an ordered quadruple of points on $C^{\prime}$, and let $g$ and $h$ be Möbius transformations with $g\left(z_{1}\right)=\infty$ and $\left.h^{\prime} z_{1}^{\prime}\right)=\infty$. Then $h \circ f \circ g^{-1} \in Q_{K}$ in $R^{2}$ and it maps $g\left(z_{i}\right)$ onto $h\left(z_{i}^{\prime}\right)$. Since $z_{1}, z_{2}, z_{3}, z_{4}$ is an ordered quadruple of points on $C$ and since $g$ maps $C$ onto a line,

$$
\left|g\left(z_{1}\right)-g\left(z_{2}\right)\right|+\left|g\left(z_{2}\right)-g\left(z_{3}\right)\right|=\left|g\left(z_{1}\right)-g\left(z_{3}\right)\right|,
$$

and Theorem 2 of [2] implies that 


$$
\sin \left(\frac{1}{2} \arcsin \left(\frac{\left|h\left(z_{1}^{\prime}\right)-h\left(z_{3}^{\prime}\right)\right|}{\left|h\left(z_{1}^{\prime}\right)-h\left(z_{2}^{\prime}\right)\right|+\left|h\left(z_{2}^{\prime}\right)-h\left(z_{3}^{\prime}\right)\right|}\right)\right) \geq \mu^{-1}\left(\frac{K \pi}{2}\right) .
$$

Because cross ratios are preserved under $h,(2)$ implies that

$$
\frac{\left|z_{1}^{\prime}-z_{2}^{\prime}\right|\left|z_{3}^{\prime}-z_{4}^{\prime}\right|+\left|z_{2}^{\prime}-z_{3}^{\prime}\right|\left|z_{4}^{\prime}-z_{1}^{\prime}\right|}{\left|z_{1}^{\prime}-z_{3}^{\prime}\right|\left|z_{2}^{\prime}-z_{4}^{\prime}\right|} \leq \csc \left(2 \arcsin \mu^{-1}\left(\frac{K \pi}{2}\right)\right),
$$

and (1) follows. For each $K$ and $C$ there exists an $f \in Q_{K}$ for which (1) holds with equality. Hence inequality (1) is sharp.

We prove next that if $D$ and $D^{\prime}$ are arbitrary and if for some constant $K$, $\frac{3 \pi}{4} \leq K<\infty$,

$$
k\left(C^{\prime}\right) \leq \frac{4 K}{3 \pi}
$$

for all circles $C \subset D$, then $f \in Q_{K}$. We do this in two steps.

We observe first that for each Jordan curve $C \subset R^{2}$,

$$
d(C)^{2} \leq 3 k(C) a(C),
$$

where $d(C)$ and $a(C)$ denote the diameter and area, respectively, of the bounded component $\Delta$ of the complement of $C$. To see this, choose $z_{1}, z_{3} \in C$ so that $\left|z_{1}-z_{3}\right|=d(C)$; by making a preliminary change of variable, we may assume that $z_{1}=0$ and $z_{3}=d(C)$. Next for each $x, 0<x<d(C)$, choose $z_{2}, z_{4} \equiv C$ so that $z_{1}, z_{2}, z_{3}, z_{4}$ is an ordered quadruple of points on $C$ and so that the open segment joining $z_{2}$ and $z_{1}$ lies in the intersection of $\Delta$ and the vertical line through $z=x$. Then

$$
2 x(d(C)-x) \leq\left|z_{1}-z_{2}\right|\left|z_{3}-z_{4}\right|+\left|z_{2}-z_{2}\right|\left|z_{4}-z_{1}\right| \leq k(C) d(C)\left|z_{2}-z_{4}\right|,
$$

and if we integrate this inequality from 0 to $d(C)$, we obtain (4).

Next from (3) and (4) it follows that

$$
d\left(C^{\prime}\right)^{2} \leq \frac{4 K}{\pi} a\left(C^{\prime}\right)
$$

for each circle $C \subset D$. Using (5), one can show by means of a familiar lengtharea argument that $f$ is $A C L$ in $D$ (See, for example, Step 1 in the proof of Theorem 2 in [11].). Then $f$ is differentiable a.e. in $D$ by [14], (5) implies that $\left|f_{z}\right|+\left|f_{\bar{z}}\right| \leq K\left(\left|f_{z}\right|-\left|f_{\bar{z}}\right|\right)$ at each point of differentiability, and hence $f \in Q_{K}$.

The desired equivalence is now an immediate consequence of what we have proved above. 


\section{REFERENCES}

[1] S. B. Agard, Topics in the theory of quasiconformal mappings, University of Michigan dissertation, 1965.

[2] S. B. Agard and F. W. Gehring. Angles and quasiconformal mappings, Proc. London Math. Soc. (3) 14 A (1965), pp. 1-21.

[3] L. V. Ahlfors, Conformal mapping, Lecture notes transcribed by R. Osserman at Oklahoma A. and M. College, 1951.

[4] L. V. Ahlfors, On quasiconformal mappings, J. d'Analyse Math. 3 (1954), pp. 1-58.

[5] L. V. Ahlfors, Quasiconformal reflections, Acta Math. 109 (1963), pp. 291-301.

[6] L. V. Ahlfors, Extension of quasiconformal mappings from two to three dimensions, Proc. Nat. Acad. Sci. USA 51 (1964), pp. 768-771.

[7] L. V. Ahlfors and L. Bers, Riemann's mapping theorem for variable metrics, Ann. Math. 72 (1960), pp. 385-404.

[8] L. Bers, On a theorem of Mori and the definition of quasiconformality, Trans. Amer. Math. Soc. 84 (1957), pp. 78-84.

[9] L. Bers, The equivalence of two definitions of quasiconformal mappings, Comm. Math. Helv. 37 (1962), pp. 148-154.

[10] A. Beurling and L. V. Ahlfors, The boundary correspondence under quasiconformal mappings, Acta Math. 96 (1956), pp. 125-142.

[11] F. W. Gehring, The definitions and exceptional sets for quasiconformal mappings, Ann. Acad. Sci. Fenn. 281 (1960), pp. 1-28.

[12] F. W. Gehring, Rings and quasiconformal mappings in space, Trans. Amer. Math. Soc. 103 (1962), pp. 353-393.

[13] F. W. Gehring, Extensicn of quasiconformal mappings in three space, J. d'Analyse Math. 14 (1965), pp. 171-182.

[14] F. W. Gebring and O. Lehto, On the total differentiability of functions of a complex variable, Ann. Acad. Sci. Fenn. 272 (1959), pp. 1-9.

[15] F. W. Gehring and J. Väisälä, On the geometric definition for quasiconformal mappings, Comm. Math. Helv. 36 (1961), pp. 19-32.

[16] J. Hersch, Longueurs extremales et theorie des fonctions, Comm. Math. Helv. 29 (1955), pp. 301-337.

[17] J. A. Kelingos, Characterizations of quasiconformal mappings in terms of harmonic and hyperbolic measure, Ann. Acad. Sci. Fenn. 368 (1965), pp. 1-16.

[18] O. Lehto and K. I. Virtanen, Quasikonforme Abbildungen, Springer-Verlag, BerlinHeidelberg-New York 1965.

[19] C. Loewner, On the conformal capacity in space. J. Math. Mech. 8 (1959), pp. 411-414.

[20] A. Mori, On quasi-conformality and pseudo-analyticity, Trans. Amer. Math. Soc. 84 (1957), pp. 56-77.

[21] I. N. Pesin, Metric properties of Q-quasiconformal mappings, Mat. Sbornik 40 (82)(1956), pp. 281-294 (Russian).

[22] A. Pfluger, Quasikonforme Abbildungen und logarithmische Kapazität, Ann. Inst. Fourier Grenoble 2 (1951). pp. 69-80.

[23] A. Pfluger, Über die Äquivalenz der geometrischen und der analytischen Definition quasikonformer Abbildungen, Comm. Math. Helv. 33 (1959), pp. 23-33.

[24] E. Reich, On a characterization of quasiconformal mappings, Comm. Math. Helv. 37 
(1962), pp. 44-48.

[25] H. Renggli, Quasiconformal mappings and extremal lengths, Amer. J. Math. 86 (1964), pp. 63-69.

[26] O. Taari, Charakterisierung der Quasikonformität mit Hilfe der Winkelverzerrung, Ann. Acad. Sci. Fenn. 390 (1966), pp. 1-43.

[27] J. Väisälä, On quasiconformal mappings in space, Ann. Acad. Sci. Fenn. 298 (1961), pp. 1-36.

University of Michigan

Ann Arbor, Michigan

Harvard University

Cambridge Massachusetts 